\title{
On some geometric properties of Banach spaces of continuous functions on separable compact lines by
}

\author{
Artur MICHALAK \\ Presented by Stanistaw KWAPIEŃ
}

\begin{abstract}
Summary. We study properties of Banach spaces $C(L)$ of all continuous scalar (real or complex) functions on compact lines $L$. First we show that if $L$ is a separable compact line, then for every closed linear subspace $X$ of $C(L)$ with separable dual the quotient space $C(L) / X$ possesses a sequence of continuous linear functionals separating its points. Next we show that for any compact line $L$ the space $C(L)$ contains no subspace isomorphic to a $C(K)$ space where $K$ is a separable nonmetrizable scattered compact Hausdorff space with countable height.
\end{abstract}

1. Introduction. A totally (= linearly) ordered set equipped with the order topology which is a compact space is called a compact line. We investigate Banach spaces $C(L)$ of all continuous scalar functions on compact lines $L$. Properties of those spaces have been studied by many authors. Locally uniformly convex and Kadec renorming of $C(L)$ was investigated in [7] and [8]. Properties of real continuous functions on scattered compact lines were studied in [11]. Correa and Tausk [2] showed that for any compact line $L$ each subspace of $C(L)$ isomorphic to $c_{0}$ is complemented.

The present paper is devoted to showing that for every separable compact line $L$ and for every closed linear subspace $X$ of $C(L)$ with separable dual the quotient space $C(L) / X$ possesses a sequence of continuous linear functionals separating its points and in consequence:

(1) $C(L) / X$ has no subspace isomorphic to a nonseparable WCG Banach space,

2010 Mathematics Subject Classification: Primary 46B20; Secondary 46E15, 54F05.

Key words and phrases: compact lines, Banach spaces of continuous functions.

Received 5 October 2016; revised 19 April 2017.

Published online 5 June 2017. 
(2) every weakly compact subset of $C(L) / X$ is separable,

(3) $(C(L) / X)^{*}$ is separable in the *weak topology,

(4) for any compact line $M$ the space $C(M)$ contains no subspace isomorphic to a $C(K)$ space where $K$ is a separable nonmetrizable scattered compact Hausdorff space with countable height.

The assumption of separability of $L$ cannot be omitted in our theorem. For every nonseparable compact line $L$ and for every closed separable linear subspace $X$ of $C(L)$ the quotient space $C(L) / X$ does not possess a sequence of continuous linear functionals separating points. This follows from the fact that every Radon measure on any compact line has separable support. If a compact Hausdorff space $K$ is separable, then the evaluation functionals at points of a countable dense subset of $K$ form a countable set of continuous linear functionals separating the points of $C(K)$. However it is not true in general that a quotient space $C(K) / X$ has the same property. For instance, if $K=\mathbb{L}$, the two-arrows space, then $K$ is a separable compact line, $C(K)$ is isometrically isomorphic to the Banach space $D(0,1)$, and $D(0,1) / C([0,1])$ does not possess a sequence of continuous linear functionals separating its points. The latter is a consequence of the fact that $D(0,1) / C([0,1])$ is isomorphic to $c_{0}([0,1])$ (see [3, Example 2]). Recall that the Banach space $D(0,1)$ consists of all scalar functions on $[0,1]$ that are right continuous at each point of $[0,1)$ and left continuous at 1 with a left-hand limit at each point of $(0,1]$, and it is equipped with the supremum norm. The present author [10] studied the properties of the $c_{0^{-}}$ sum $\left(\bigoplus_{n=2}^{\infty} C\left(\mathbb{L}^{n}\right)\right)_{c_{0}}$. Our main result for $C(\mathbb{L})$ may be deduced from [10, Thm. 3.1].

The paper is divided into three sections. Properties of compact lines and continuous functions on these spaces are gathered in the first part of the second section. Properties of countable intersections of hyperplanes in Banach spaces are investigated in the second part of that section. The main results of the paper are presented in the third section.

2. Preliminaries. The Banach space of all continuous scalar (real or complex) functions on a compact Hausdorff space $K$ with the supremum norm is denoted by $C(K)$. The topological dual of a Banach space $X$ is denoted by $X^{*}$. For a subset $Y$ of a Banach space $X$, let $Y^{\perp}$ denote the subspace $\left\{x^{*} \in X^{*}: x^{*}(Y)=0\right\}$ of $X^{*}$. The linear hull, the closed hull in the norm topology and the closed hull in the *weak topology of a subset $A$ of a Banach space $X$ are denoted by $\operatorname{lin}(A), \bar{A}$ and $\bar{A}^{* w}$, respectively. For a nonempty set $\Gamma$, the Banach space (under the supremum norm) of all scalar functions $f$ on $\Gamma$ such that for every $\eta>0$ the set $\{\gamma \in \Gamma:|f(\gamma)|>\eta\}$ is finite is denoted by $c_{0}(\Gamma)$. The Banach space of all scalar bounded sequences 
equipped with the supremum norm is denoted by $l_{\infty}$. The cardinality of a set $A$ is denoted by $\operatorname{card}(A)$.

Let $L$ be a compact line. For every $v \in L \backslash\{\min (L)\}, w \in L \backslash\{\max (L)\}$ and $s, t \in L$ with $s<t$ the sets $\{u \in L: u<v\}=[\min (L), v),\{u \in L$ : $u>w\}=(w, \max (L)]$ and $(s, t)=\{u \in L: s<u<t\}$ are called open intervals. The family of open intervals forms a base of the topology of $L$. Every nonempty closed subset of $L$ has the minimum and maximum, which are members of the set (see [6] or [4, p. 53]). The closure of a subset $A$ of $L$ is denoted by $\bar{A}$. For every $u \in L$, we denote

$$
u_{-}=\max \overline{\{v \in K: v<u\}} \text { and } \quad u_{+}=\min \overline{\{v \in K: v>u\}} \text {. }
$$

We set $\min (L)_{-}=\min (L)$ and $\max (L)_{+}=\max (L)$. Note that if for some $t \in L$ we have $t_{-} \neq t$, then the interval $\left(t_{-}, t\right)$ is an empty set and $\left(t_{-}\right)_{+}=t$. Similarly if $t_{+} \neq t$, then $\left(t, t_{+}\right)=\emptyset$ and $\left(t_{+}\right)_{-}=t$.

Proposition 2.1. Let $L$ be a compact line.

(a) For every continuous function $f: L \rightarrow \mathbb{C}$ and $\varepsilon>0$ the set

$$
\left\{t \in L:\left|f\left(t_{+}\right)-f(t)\right|>\varepsilon\right\}
$$

is finite.

(b) If the set $\left\{t \in L: t_{+} \neq t\right\}$ is uncountable, then $L$ is nonmetrizable.

(c) If there exists a subset $A$ of $L$ such that $\operatorname{card}(\bar{A})>\operatorname{card}(A)$, then

(1) there exists a nondecreasing continuous surjection $f: L \rightarrow[0,1]$,

(2) there exists a countable subset $B$ of $L$ with $\bar{B}$ uncountable.

Parts (a) and (b) are well known. Part (c) follows from [11, Thm. 1] and considerations in that paper. We present a short proof of (c) for completeness.

Proof of (c). It is clear that $A$ is infinite. Suppose that $\operatorname{card}(A)=\gamma$. Let

$$
\Pi=\left\{k / 2^{n}: 0 \leq k \leq 2^{n}, k, n \in \mathbb{N} \cup\{0\}\right\} .
$$

First we show that for every $a \in \Pi$ there exists $t_{a} \in \bar{A}$ such that $t_{a}<t_{b}$ if $a<b$. Let

$$
\begin{aligned}
& D=\{s \in \bar{A}: \operatorname{card}([\min (L), s) \cap \bar{A}) \leq \gamma\}, \\
& E=\{s \in \bar{A}: \operatorname{card}((s, \max (L)] \cap \bar{A}) \leq \gamma\} .
\end{aligned}
$$

Let $v=\max \bar{D}$ and $w=\min \bar{E}$. It is clear that $v, w \in \bar{A}$. By the Hessenberg theorem $(\gamma \cdot \gamma=\gamma)$ we have

$$
\operatorname{card}\left(\bigcup_{s \in A, s<v}[\min (L), s) \cap \bar{A}\right) \leq \gamma, \quad \operatorname{card}\left(\bigcup_{s \in A, s>w}(s, \max (L)] \cap \bar{A}\right) \leq \gamma
$$

If $\max \overline{\{s \in A: s<v\}}<v$, then $v \in D$. If $\max \overline{\{s \in A: s<v\}}=v$, then $[\min (L), v) \cap \bar{A}=\bigcup_{s \in A, s<v}[\min (L), s) \cap \bar{A}$ and also $v \in D$. If 
$\min \overline{\{s \in A: s>w\}}>w$, then $w \in E$. If $\min \overline{\{s \in A: s>w\}}=w$, then $(w, \max (L)] \cap \bar{A}=\bigcup_{s \in A, s>w}(s, \max (L)] \cap \bar{A}$ and also $w \in E$. Since $\operatorname{card}(\bar{A})>\gamma$, for every $u \in(v, w) \cap \bar{A}$ we have $\operatorname{card}((v, u) \cap \bar{A})>\gamma$ and $\operatorname{card}((u, w) \cap \bar{A})>\gamma$. We set $t_{0}=v$ and $t_{1}=w$. Let $t_{1 / 2}$ be any element of $(v, w) \cap \bar{A}$. Continuing the procedure we find a set $\left\{t_{a}: a \in \Pi\right\}$ with the desired property. Let

$$
f(x)= \begin{cases}\inf \left\{a \in \Pi: x \in\left[\min (L), t_{a}\right)\right\} & \text { if } x<t_{1}, \\ 1 & \text { if } x \geq t_{1} .\end{cases}
$$

By the Urysohn Lemma (see [4, p. 43]) the function $f$ is continuous. For every $a \in \Pi$, we have $t_{a} \in \bigcap_{b \in \Pi, b>a}\left[\min (L), t_{b}\right)$ and $t_{a} \notin \bigcup_{b \in \Pi, b \leq a}\left[\min (L), t_{b}\right)$. Hence $f\left(t_{a}\right)=a$ for every $a \in \Pi$. Since $L$ is a compact space, $f(L)=[0,1]$. It is clear that $\overline{\left\{t_{a}: a \in \Pi\right\}}$ has cardinality continuum.

The support of a Radon measure $\mu$ on a compact Hausdorff space $K$ is the smallest closed subset $M$ of $K$ such that $|\mu|(K \backslash M)=0$ where $|\mu|$ is the variation of $\mu$.

Proposition 2.2. Let $L$ be a compact line.

(a) Every Radon measure on $L$ has separable support.

(b) If $X$ is a closed linear subspace of $C(L)$ which is isomorphic to a subspace of $l_{\infty}$, then there exists a separable and closed subset $M$ of $L$ such that the restriction operator $R: C(L) \rightarrow C(M)$ given by the formula

$$
R(f)=\left.f\right|_{M} \quad \text { for every } f \in C(L)
$$

is an isomorphism on $X$.

Part (a) is known but for completeness we present its proof.

Proof. (a) It is enough to show that any probability Radon measure $\mu$ on $L$ with $\mu(\{t\})=0$ for every $t \in L$ has separable support. Suppose that $\mu$ has these properties. Let $f: L \rightarrow[0,1]$ be given by

$$
f(t)=\mu([\min (L), t]) .
$$

It is clear that $f$ is a nondecreasing function and $\{0,1\} \subset f(L)$.

Let $a \in(0,1)$. Suppose that $a \notin f(L)$. Let $A=f^{-1}([0, a))$ and $B=$ $f^{-1}((a, 1])$. Then $A \cup B=L$. Let $v=\max \bar{A}$. If $v \in A$, then $A=[\min (L), v]$. If $v \notin A$, then $v \in B$ and $A=[\min (L), v)$. Indeed, otherwise there exists $t \in B$ such that $t<v$; since $v \in \bar{A}$, the set $(t, v) \cap A$ is nonempty; since $f$ is nondecreasing, $f(t)<a$, a contradiction.

This shows that either $A=[\min (L), v]$ and $B=(v, \max (L)]$, or $A=$ $[\min (L), v)$ and $B=[v, \max (L)]$. Consider the first case. For every compact subset $K$ of $B$ we have $K \subset[\min (K), \max (L)] \subset B$ and $\mu(K) \leq 1-a$. Since $\mu$ is a Radon measure, we have

$$
\mu(B)=\sup \{\mu(K): K \subset B, K \text { compact }\} \leq 1-a .
$$


Hence

$$
1=\mu(L)=\mu(A)+\mu(B)<a+1-a=1,
$$

a contradiction. This shows that $f(L)=[0,1]$. Considerations in the second case are similar.

For every $q \in \mathbb{Q} \cap[0,1]$, let $t_{q} \in L$ be such that $f\left(t_{q}\right)=q$. Let $K=$ $\left\{t_{q}: q \in \mathbb{Q} \cap[0,1]\right\}$. The set $L \backslash K$ is a union of open intervals with endpoints in $K$. Let $\left(t_{1}, t_{2}\right) \subset L \backslash K$ be an interval such that $t_{1}, t_{2} \in K$. If $\mu\left(\left(t_{1}, t_{2}\right)\right)>$ $\varepsilon>0$, then $\left(f\left(t_{1}\right), f\left(t_{1}\right)+\varepsilon\right) \not \subset f(L)$. This shows that $\mu\left(\left(t_{1}, t_{2}\right)\right)=0$. Since $\mu$ is a Radon measure, $\mu(L \backslash K)=0$.

Part (b) is a straightforward consequence of (a).

The most important example of a separable nonmetrizable compact line is the two-arrows space $\mathbb{L}=\{(x, 0): 0<x \leq 1\} \cup\{(x, 1): 0 \leq x<1\}$. The order on $\mathbb{L}$ is defined by $(x, p)<(y, r)$ if either $x<y$, or $x=y$ and $p<r$. Ostaszewski [12] showed that a totally ordered set $K$ equipped with the order topology is compact and separable if and only if $K$ is order-isomorphic to a subset $L$ of $\mathbb{L}$ such that $\{s \in[0,1]:(s, 0) \in L\}$ is a closed subset of $[0,1]$, and $(s, 0) \in L$ whenever $(s, 1) \in L$.

We will need the following well known facts on separable compact lines.

Proposition 2.3. Let $L$ be a separable compact line.

(a) $L$ is first countable.

(b) If $X$ is a subset of $C(L)$ such that the set

$$
U=\left\{t \in L \text { : there exists } f \in X \text { such that } f\left(t_{+}\right) \neq f(t)\right\}
$$

is countable, then $X$ is separable.

(c) The linear operator $J: C(L) \rightarrow c_{0}(L)$ given by the formula

$$
J(f)(t)=f\left(t_{+}\right)-f(t) \quad \text { for every } t \in L
$$

is a well defined continuous operator with separable kernel.

(d) If $L$ is uncountable, then there exists a nondecreasing continuous surjection $f: L \rightarrow[0,1]$.

Proof. Let $Q_{0}$ be a countably dense subset of $L$ such that

$$
\{\min (L), \max (L)\} \subset Q_{0} .
$$

Let $Q=\bigcup_{t \in Q_{0}}\left\{t_{-}, t, t_{+}\right\}$.

(a) Let $t \in L$. If $t_{-}=t=t_{+}$, then $\{(s, u): s, u \in Q, s<t<u\}$ is a base of open neighbourhoods of $t$. If $t_{-}=t<t_{+}$, then $\{(s, t]: s \in Q, s<t\}$ is a base of open neighbourhoods of $t$. If $t_{-}<t=t_{+}$, then $\{[t, s): s \in Q, s>t\}$ is a base of open neighbourhoods of $t$. If $t_{-}<t<t_{+}$, then $\{\{t\}\}$ is a base of open neighbourhoods of $t$.

(b) Since $L$ is a compact Hausdorff space, for every $s, u \in Q$ with $s<u$ there exists $f_{s, u} \in C(L)$ such that $f_{s, u}(L) \subset[0,1], f_{s, u}([\min (L), s])=\{0\}$ 
and $f_{s, u}([u, \max (L)])=\{1\}$. For every $s \in U$, let $g_{s}=\chi_{\left[s_{+}, \max (L)\right]}$. It is clear that $\left[s_{+}, \max (L)\right]$ is open and closed. Hence $g_{s}$ is continuous for every $s \in U$. Let

$$
F=\left\{f_{s, u}: s, u \in Q, s<u\right\} \cup\left\{g_{s}: s \in U\right\} .
$$

Let $\mathcal{R}$ be an equivalence relation on $L$ such that $v \mathcal{R} w$ if $f(v)=f(w)$ for every $f \in F$. Let $L_{0}=L / \mathcal{R}$ with the quotient topology. It is clear that $L_{0}$ is Hausdorff, compact and metrizable. Let $\mathfrak{q}: L \rightarrow L_{0}$ be the quotient map.

Suppose that $f \in X$ and there exist $v, w \in L$ such that $v<w$ and $v \mathcal{R} w$ and $f(v) \neq f(w)$.

If the interval $(v, w)$ contains at least two members of $Q$, then there exist $s, u \in Q$ such that $s, u \in(v, w), s<u, f_{s, u}(v)=0$ and $f_{s, u}(w)=1$. Hence $v$ and $w$ are not $\mathcal{R}$-related. If $(v, w)$ contains exactly one point $s \in Q$, then $w=s_{+} \in Q, f_{s, w}(v)=0$ and $f_{s, w}(w)=1$. If $(v, w)=\emptyset$, then $w=v_{+}$, $v \in U, g_{v}(v)=0$ and $g_{v}(w)=1$.

Thus in each case above we have arrived at a contradiction. This shows that there exists $h \in C\left(L_{0}\right)$ such that $f=h \circ \mathfrak{q}$. Since $L_{0}$ is compact and metrizable, $X$ is a subset of the separable subspace $\left\{h \circ \mathfrak{q}: h \in C\left(L_{0}\right)\right\}$ of $C(L)$.

(c) By Proposition 2.1(a) the operator $J$ is well defined. It is easy to check that $J$ is continuous. The separability of ker $J$ easily follows from (b).

(d) This part follows from Proposition 2.1(c).

We will need the following well known facts.

Proposition 2.4.

(a) For a closed linear subspace $Y$ of a Banach space $X$ the following conditions are equivalent:

(1) there exists a sequence $\left(x_{n}^{*}\right) \subset X^{*}$ such that $Y=\bigcap_{n=1}^{\infty} \operatorname{ker} x_{n}^{*}$,

(2) there exists a sequence $\left(x_{n}^{*}\right) \subset(X / Y)^{*}=Y^{\perp}$ separating the points of $X / Y$.

(b) If $X$ is a separable Banach space, then for every closed linear subspace $Y$ of $X$ there exists a sequence $\left(x_{n}^{*}\right) \subset X^{*}$ such that $Y=\bigcap_{n=1}^{\infty} \operatorname{ker} x_{n}^{*}$.

$\left(\mathrm{b}^{\prime}\right)$ If $Y$ is a closed linear subspace of a Banach space $X$ such that $X / Y$ is separable, then there exists a sequence $\left(x_{n}^{*}\right) \subset X^{*}$ such that $Y=$ $\bigcap_{n=1}^{\infty} \operatorname{ker} x_{n}^{*}$.

(c) If for a Banach space $X$ there exists a sequence $\left(x_{n}^{*}\right) \subset X^{*}$ separating the points of $X$, then there exists an injective continuous linear operator $S: X \rightarrow l_{\infty}$.

$\left(c^{\prime}\right)$ If there exists a separable closed linear subspace $Y$ of a Banach space $X$ such that $Y=\bigcap_{n=1}^{\infty} \operatorname{ker} x_{n}^{*}$ for some sequence $\left(x_{n}^{*}\right) \subset X^{*}$, then there exists an injective continuous linear operator $S: X \rightarrow l_{\infty}$. 
(d) If $K$ is a nonseparable compact Hausdorff space such that every Radon measure on $K$ has separable support, then for any sequence $\left(x_{n}^{*}\right) \subset$ $C(K)^{*}$ the subspace $Y=\bigcap_{n=1}^{\infty} \operatorname{ker} x_{n}^{*}$ of $C(K)$ is nonseparable.

(e) If for a Banach space $X$ there exists a sequence $\left(x_{n}^{*}\right) \subset X^{*}$ separating the points of $X$ and $P: X \rightarrow X$ is a continuous projection, then $P(X)=\bigcap_{n=1}^{\infty} \operatorname{ker}(I-P)^{*}\left(x_{n}^{*}\right)$.

Proof. Parts (a), (b), (b'), (d) and (e) are obvious. Part (c) easily follows from $\left(c^{\prime}\right)$.

$\left(\mathrm{c}^{\prime}\right)$ Let $\left(x_{n}^{*}\right) \subset X^{*}$ be a sequence such that $Y=\bigcap_{n=1}^{\infty} \operatorname{ker} x_{n}^{*}$ and $\left\|x_{n}^{*}\right\|=1$ for every $n$. Since $Y$ is separable, there exists a sequence $\left(y_{n}^{*}\right) \subset Y^{*}$ separating the points of $Y$ and such that $\left\|y_{n}^{*}\right\|=1$ for every $n$. For every $n$, let $\tilde{y}_{n}^{*} \in X^{*}$ be an extension of $y_{n}^{*}$ with $\left\|\tilde{y}_{n}^{*}\right\|=\left\|y_{n}^{*}\right\|$. It is easy to check that the linear operator $S: X \rightarrow l_{\infty}$ given by the formula $S(x)=\left(z_{n}^{*}(x)\right)$ is continuous and injective where $z_{2 n-1}^{*}=x_{n}^{*}$ and $z_{2 n}^{*}=\tilde{y}_{n}^{*}$ for every $n$.

The Johnson-Lindenstrauss space $J L_{2}$ (see [9]) is an example of a Banach space $X$, not isomorphic to a subspace of $l_{\infty}$, which possesses a sequence $\left(x_{n}^{*}\right) \subset X^{*}$ separating the points of $X$. A Banach space $X$ is called weakly compactly generated (WCG) if there exists a weakly compact subset $A$ of $X$ such that the linear hull of $A$ is dense in $X$ in the norm topology. We refer to [15] for more information about this class. For any nonempty set $\Gamma$ the space $c_{0}(\Gamma)$ is a WCG Banach space. The spaces $C(\mathbb{L})$ and $l_{\infty}$ are not WCG. We will need the following property of WCG Banach spaces.

Proposition 2.5. If $X$ is a $W C G$ Banach space and $Z$ is a Banach space, and $S: X \rightarrow Z$ is a continuous linear operator such that $S(X)$ and $\operatorname{ker}(S)$ are separable subspaces of $Z$ and $X$, respectively, then $X$ is separable.

Proof. Let $A$ be a weakly compact subset of $X$ whose linear hull is dense in $X$. Let $B$ be a countable and dense subset of $S(A)$. Let $C$ be a countable subset of $A$ such that $S(C)=B$. Let $D$ be a countable and dense subset of $\operatorname{ker}(S)$. Let $x \in A$. We find a sequence $\left(v_{n}\right) \subset C$ such that $\left(S\left(v_{n}\right)\right)$ converges to $S(x)$ in the norm topology. By the Eberlein-Šmulian theorem there exists a subsequence $\left(v_{k_{n}}\right)$ of $\left(v_{n}\right)$ that weakly converges to $y \in A$. It is clear that $x-y \in \operatorname{ker}(S)$. Then there exists a sequence $\left(w_{n}\right)$ in $D$ such that $x-y=\lim _{n \rightarrow \infty} w_{n}$. Therefore $\left(v_{k_{n}}+w_{n}\right)$ converges to $x$ in the weak topology. Thus we have shown that the closed linear hull of $C+D$ contains $A$. Therefore $A$ and $X$ are separable in the norm topology.

Corollary 2.6.

(a) If $X$ is a nonseparable WCG Banach space, then for any sequence $\left(x_{n}^{*}\right) \subset X^{*}$ the subspace $Y=\bigcap_{n=1}^{\infty} \operatorname{ker} x_{n}^{*}$ of $X$ is nonseparable.

(b) If $L$ is a nonseparable compact line, then for any sequence $\left(x_{n}^{*}\right) \subset C(L)^{*}$ the subspace $Y=\bigcap_{n=1}^{\infty} \operatorname{ker} x_{n}^{*}$ of $C(L)$ is nonseparable. 
Proof. (a) Every weakly compact subset of $l_{\infty}$ is separable in the norm topology. Therefore (a) is a straightforward consequence of Propositions 2.4 ( $\left.\mathrm{c}^{\prime}\right)$ and 2.5.

Part (b) is a consequence of Propositions 2.2(a) and 2.4(d).

The class of nonseparable compact Hausdorff spaces $K$ such that every Radon measure on $K$ has separable support is quite wide. It contains for example all nonseparable Rosenthal compacts (see [5]).

3. Main results. Now we are ready to state our main result.

TheOREM 3.1. Let $L$ be a separable compact line. For a closed linear subspace $X$ of $C(L)$ such that $X^{*}$ is separable there exists a sequence $\left(x_{n}^{*}\right) \subset C(L)^{*}$ such that $X=\bigcap_{n=1}^{\infty} \operatorname{ker} x_{n}^{*}$.

Proof. Let $Q_{0}$ be a countably dense subset of $L$ with $\{\min (L), \max (L)\}$ $\subset Q_{0}$. Let $Q_{1}=\bigcup_{t \in Q_{0}}\left\{t_{-}, t, t_{+}\right\}$. Since $X$ is a separable subspace of $C(L)$, there exists a countable subset $Q_{2}$ of $L$ such that $Q_{1} \subset Q_{2}$ and $J(X) \subset$ $\overline{\operatorname{lin}}\left\{e_{t}: t \in Q_{2}\right\}$ where $J$ is the operator defined in Proposition 2.3 and $e_{t}$ is the $t$ th unit vector in $c_{0}(L)$. For every $t \in L \backslash Q_{2}$ we have the equality

$$
\delta_{t_{+}}+X^{\perp}=\delta_{t}+X^{\perp} \in C(L)^{*} / X^{\perp}
$$

where $\delta_{u}$ is the Dirac measure at $u$. The quotient space $C(L)^{*} / X^{\perp}$ is isometric to $X^{*}$. Hence it is separable. By the Bartle and Graves theorem (see [1, Thm. 4]) there exists a continuous map $S: C(L)^{*} / X^{\perp} \rightarrow C(L)^{*}$ such that $\mathfrak{q} \circ S(x)=x$ and $\|S(x)\| \leq 2\|x\|$ for every $x \in C(L)^{*} / X^{\perp}$, where $\mathfrak{q}: C(L)^{*} \rightarrow C(L)^{*} / X^{\perp}$ is the quotient map. Hence the subset $\left\{S\left(\delta_{t}+X^{\perp}\right): t \in L\right\}$ of $C(L)^{*}$ is separable in the norm topology.

For every $a, b \in Q_{2}$ with $a<b$, let $T_{a, b}$ be a countable subset of $[a, b]$ such that

$$
\left\{S\left(\delta_{t}+X^{\perp}\right): t \in[a, b]\right\} \subset \overline{\left\{S\left(\delta_{t}+X^{\perp}\right): t \in T_{a, b}\right\}} .
$$

Since $C(L)^{*} / X^{\perp}$ is separable, such sets exist. Let

$$
T=\bigcup_{a, b \in Q_{2}, a<b} T_{a, b} .
$$

For every $\sigma>0$, let $A_{\sigma}$ be the subset of all $t \in\left\{s \in L: s=s_{+}\right\}$such that there exists $u_{t} \in Q_{2}$ such that $u_{t}>t$ and

$$
\left\|S\left(\delta_{t}+X^{\perp}\right)-S\left(\delta_{s}+X^{\perp}\right)\right\|>\sigma
$$

for every $s \in\left(t, u_{t}\right)$.

Suppose that $A_{\sigma}$ is uncountable. Then there exists $u \in Q_{2}$ and an uncountable subset $C$ of $A_{\sigma}$ such that $u_{t}=u$ for every $t \in C$. It is clear that for every $s, t \in C$ with $s \neq t$ we have

$$
\left\|S\left(\delta_{t}+X^{\perp}\right)-S\left(\delta_{s}+X^{\perp}\right)\right\|>\sigma .
$$


Since $\left\{S\left(\delta_{t}+X^{\perp}\right): t \in L\right\}$ is a separable subset of the metric space $C(L)^{*}$, this is impossible. Therefore $A_{\sigma}$ is countable for every $\sigma>0$. Hence also $\bigcup_{\sigma>0} A_{\sigma}$ is countable.

For every $\sigma>0$, let $B_{\sigma}$ be the subset of all $t \in\left\{s \in L: s=s_{-}\right\}$such that there exists $v_{t} \in Q_{2}$ such that $v_{t}<t$ and

$$
\left\|S\left(\delta_{t}+X^{\perp}\right)-S\left(\delta_{s}+X^{\perp}\right)\right\|>\sigma
$$

for every $s \in\left(v_{t}, t\right)$. It is clear that also $\bigcup_{\sigma>0} B_{\sigma}$ is countable. Let $Q_{3}=$ $Q_{2} \cup\left(\bigcup_{\sigma>0} B_{\sigma}\right) \cup\left\{t \in L: t_{+} \in \bigcup_{\sigma>0} A_{\sigma}\right\}$. Note that

$$
\begin{aligned}
& \left\{t \in L: t \neq t_{+}\right\} \subset\left\{t \in L: t=t_{-}, t \neq t_{+}\right\} \cup Q_{1} \\
& \subset\left\{t \in L \backslash Q_{3}: t=t_{-}, t \neq t_{+},\left(t_{+}\right)_{+}=t_{+}\right\} \cup Q_{3} \cup\left\{t \in L: t_{+} \in Q_{1}\right\} .
\end{aligned}
$$

For every $t \in\left\{s \in L: s=s_{-}, s \neq s_{+},\left(s_{+}\right)_{+}=s_{+}\right\} \backslash Q_{3}$ and $s, u \in L$ such that $s<t<t_{+}<u$ we have

$$
\begin{aligned}
S\left(\delta_{t}+X^{\perp}\right) & \in \overline{\left\{S\left(\delta_{v}+X^{\perp}\right): v \in(s, t)\right\}}, \\
S\left(\delta_{t_{+}}+X^{\perp}\right) & \in \overline{\left\{S\left(\delta_{v}+X^{\perp}\right): v \in\left(t_{+}, u\right)\right\}} .
\end{aligned}
$$

Let

$$
U=\left\{S\left(\delta_{t}+X^{\perp}\right)-\delta_{t}: t \in T\right\} .
$$

The set $U$ is a countable subset of $X^{\perp}$. Suppose that $t \in\left\{s \in L: s=s_{-}\right.$, $\left.s \neq s_{+},\left(s_{+}\right)_{+}=s_{+}\right\} \backslash Q_{3}$. By $(* *)$ and Proposition 2.3(a) there exist strictly increasing sequences $\left(a_{n}\right),\left(b_{n}\right) \subset Q_{1}$ and a sequence $\left(t_{n}\right) \subset L$ such that $a_{n}<b_{n}<a_{n+1}$ and $t_{n} \in T_{a_{n}, b_{n}}$ for every $n, \lim _{n \rightarrow \infty} a_{n}=\lim _{n \rightarrow \infty} b_{n}=t$, and

$$
S\left(\delta_{t}+X^{\perp}\right)=S\left(\delta_{t_{+}}+X^{\perp}\right)=\lim _{n \rightarrow \infty} S\left(\delta_{t_{n}}+X^{\perp}\right)
$$

where the limit is taken in the norm topology of $C(L)^{*}$. It is clear that $\left(t_{n}\right)$ converges to $t$ in $L$. Hence $\left(\delta_{t_{n}}\right)$ converges to $\delta_{t}$ in the $*$ weak topology of $C(L)^{*}$. By (**) and Proposition 2.3. (a) there exist strictly decreasing sequences $\left(c_{n}\right),\left(d_{n}\right) \subset Q_{1}$ and a sequence $\left(s_{n}\right) \subset L$ such that $c_{n}>d_{n}>c_{n+1}$ and $s_{n} \in T_{d_{n}, c_{n}}$ for every $n, \lim _{n \rightarrow \infty} c_{n}=\lim _{n \rightarrow \infty} d_{n}=t_{+}$, and

$$
S\left(\delta_{t}+X^{\perp}\right)=S\left(\delta_{t_{+}}+X^{\perp}\right)=\lim _{n \rightarrow \infty} S\left(\delta_{s_{n}}+X^{\perp}\right)
$$

where the limit is taken in the norm topology of $C(L)^{*}$. It is clear that $\left(s_{n}\right)$ converges to $t_{+}$in $L$. Hence $\left(\delta_{s_{n}}\right)$ converges to $\delta_{t_{+}}$in the $*$ weak topology of $C(L)^{*}$. Thus we have shown that

$$
S\left(\delta_{t}+X^{\perp}\right)-\delta_{t} \in \bar{U}^{* w} \quad \text { and } \quad S\left(\delta_{t_{+}}+X^{\perp}\right)-\delta_{t_{+}} \in \bar{U}^{* w}
$$

for every $t \in\left\{s \in L: s=s_{-}, s \neq s_{+},\left(s_{+}\right)_{+}=s_{+}\right\} \backslash Q_{3}$. Therefore

$$
\delta_{t}-\delta_{t_{+}} \in \bar{U}^{* w}-\bar{U}^{* w} \subset \overline{\operatorname{lin}(U)}^{* w}
$$

for every $t \in\left\{s \in L: s=s_{-}, s \neq s_{+},\left(s_{+}\right)_{+}=s_{+}\right\} \backslash Q_{3}$. 
Let $Y=\bigcap_{x^{*} \in U} \operatorname{ker} x^{*}$. Suppose that $f \in Y$. Then $y^{*}(f)=0$ for every $y^{*} \in \overline{\operatorname{lin}(U)}^{* w}$. Hence $f(t)-f\left(t_{+}\right)=0$ for every $t \in\left\{s \in L: s=s_{-}, s \neq s_{+}\right.$, $\left.\left(s_{+}\right)_{+}=s_{+}\right\} \backslash Q_{3}$. By $(*)$ and Proposition 2.3(b), $Y$ is a separable subspace of $C(L)$. An appeal to Proposition 2.4(b) completes the proof.

Let us record the following consequences of Theorem 3.1 and Corollary 2.6

Corollary 3.2. Let $L$ be a separable compact line. If $X$ is a closed linear subspace of $C(L)$ such that $X^{*}$ is separable, then

(a) $C(L) / X$ has no subspace isomorphic to a nonseparable WCG Banach space,

(b) every weakly compact subset of $C(L) / X$ is separable in the norm topology,

(c) $(C(L) / X)^{*}$ is separable in the *weak topology.

Part (a) of our next result follows from the corollary above.

Corollary 3.3. Let $L$ be a separable compact line.

(a) If a Banach space $X$ contains a closed linear subspace $Y$ such that $Y^{*}$ is separable and $X / Y$ contains a subspace isomorphic to a nonseparable $W C G$ Banach space, then $X$ is not isomorphic to a subspace of $C(L)$.

(b) If $Y$ is a closed linear subspace of a Banach space $X$ and $S: X \rightarrow C(L)$ is a continuous linear operator such that $X / Y$ is a WCG Banach space and $\overline{S(Y)}^{*}$ is separable, then $S$ has separable range.

Proof. (b) Let $\mathfrak{q}: X \rightarrow X / Y$ be the quotient map. Let $T: X / Y \rightarrow$ $C(L) / \overline{S(Y)}$ be the continuous linear operator given by the formula

$$
T(x+Y)=S(x)+\overline{S(Y)} .
$$

According to Corollary 3.2(b) the operator $T$ has separable range. Therefore so does $T \circ \mathfrak{q}$, and in consequence $\overline{S(X)} / \overline{S(Y)}$ is a separable subset of $C(L) / \overline{S(Y)}$.

A topological space $K$ is scattered if every subset $A$ of $K$ has a relatively isolated point in $A$. The derived set of $A \subset K$ is the set $A^{(1)}$ of all accumulation points of $A$ in $K$. For an ordinal number $\alpha$ we define the $\alpha$ th derived set by transfinite induction in the following way: $A^{0}=A, A^{(\alpha+1)}=\left(A^{(\alpha)}\right)^{1}$, and $A^{(\alpha)}=\bigcap_{\beta<\alpha} A^{(\beta)}$ if $\alpha$ is a limit ordinal.

The pair $(\alpha, m)$ is called the characteristic system of a scattered compact Hausdorff space $K$ if $K^{(\alpha+1)}=\emptyset$ and $K^{(\alpha)}$ is a nonempty finite set with $m$ elements (see [13]). The ordinal $\alpha$ is also called the height of $K$.

$C(K)$ spaces for scattered compact spaces $K$ were investigated by many authors (see [15]). The Johnson-Lindenstrauss space $J L_{0}$ is an example of a Banach space which is isometrically isomorphic to a $C\left(K_{1}\right)$ space where $K_{1}$ 
is a separable nonmetrizable scattered compact space such that $K_{1} \backslash K_{1}^{(1)}$ is countable, $K_{1}^{(1)} \backslash K_{1}^{(2)}$ is uncountable and $K_{1}^{(2)}$ is a singleton (see [14]). From Proposition 2.3(d) it follows that no compact line contains a subset homeomorphic to a separable nonmetrizable scattered compact space. On the other hand, if $K$ is a closed subset of a compact line $L$, then $C(K)$ is isomorphic to a subspace of $C(L)$ (see [7, Lemma 3.4]). Our next result gathers together these two facts.

COROLlary 3.4. Let $L$ be a compact line. If $K$ is a separable scattered compact Hausdorff space with characteristic system $(\alpha, m)$ where $\alpha$ is a countable ordinal, and the space $C(K)$ is isomorphic to a subspace of $C(L)$, then $K$ is countable.

Proof. Since $K$ is a separable compact Hausdorff space, the space $C(K)$ is isomorphic to a subspace of $l_{\infty}$. According to Proposition 2.2(b) the space $C(K)$ is isomorphic to a subspace of $C(M)$ where $M$ is a separable compact line.

Suppose that $K$ is uncountable. Since $K$ is separable, the set $K \backslash K^{(1)}$ is countable. Since $\alpha$ is a countable ordinal, there exists an ordinal $\beta<\alpha$ such that $K^{(\beta)} \backslash K^{(\beta+1)}$ is uncountable. Let $\gamma$ be the smallest ordinal such that $K^{(\gamma)} \backslash K^{(\gamma+1)}$ is uncountable. Let $Y=\left\{f \in C(K):\left.f\right|_{K^{(\gamma)}}=0\right\}$. It is easy to see that

$$
\|f+Y\|=\sup \left\{|f(t)|: t \in K^{(\gamma)}\right\}
$$

for every $f \in C(K)$, and the quotient space $C(K) / Y$ is isometrically isomorphic to $C\left(K^{(\gamma)}\right)$. The set $K^{(\gamma)} \backslash K^{(\gamma+1)}$ is uncountable and each of its points is isolated in $K^{(\gamma)}$. Therefore $C\left(K^{(\gamma)}\right)$ contains a subspace isomorphic to $c_{0}\left(K^{(\gamma)} \backslash K^{(\gamma+1)}\right)$. Consequently, $C(K) / Y$ contains a subspace isomorphic to a nonseparable WCG Banach space. Let $T=\left(K \backslash K^{(\gamma)}\right) \cup\{\infty\}$. Let $\mathfrak{q}: K \rightarrow T$ be given by the formula

$$
\mathfrak{q}(t)= \begin{cases}t & \text { if } t \in K \backslash K^{(\gamma)} \\ \infty & \text { if } t \in K^{(\gamma)}\end{cases}
$$

We equip $T$ with the strongest topology for which the map $\mathfrak{q}$ is continuous (the quotient topology). Since $K^{(\gamma)}$ is a closed subset of $K$, the space $T$ is Hausdorff. Now it is clear that $T$ is a compact countable scattered Hausdorff space. It is clear that $Y$ is isomorphic to a closed subspace of $C(T)$ consisting of functions that vanish at $\infty$. Therefore $Y^{*}$ is a separable space. But this contradicts Corollary 3.3(a).

\section{References}

[1] R. G. Bartle and L. M. Graves, Mappings between function spaces, Trans. Amer. Math. Soc. 72 (1952), 400-412. 
[2] C. Correa and D. V. Tausk, Compact lines and the Sobczyk property, J. Funct. Anal. 266 (2014), 5765-5778.

[3] H. H. Corson, The weak topology of a Banach space, Trans. Amer. Math. Soc. 101 (1961), 1-15.

[4] L. Gillman and M. Jerison, Rings of Continuous Functions, Van Nostrand, Princeton, NJ, 1960.

[5] G. Godefroy, Compacts de Rosenthal, Pacific J. Math. 91 (1980), 293-306.

[6] A. Haar und D. König, Über einfach geordnete Mengen, J. Reine Angew. Math. 139 (1911), 16-28.

[7] R. G. Haydon, J. E. Jayne, I. Namioka and C. A. Rogers, Continuous functions on compact totally ordered spaces that are compact in their order topologies, J. Funct. Anal. 178 (2000), 23-63.

[8] J. E. Jayne, I. Namioka and C. A. Rogers, Continuous functions on compact totally ordered spaces, J. Funct. Anal. 134 (1995), 261-280.

[9] W. B. Johnson and J. Lindenstrauss, Some remarks on weakly compactly generated Banach spaces, Israel J. Math. 17 (1974), 219-230.

[10] A. Michalak, On uncomplemented isometric copies of $c_{0}$ in spaces of continuous functions on products of the two-arrows space II, Indag. Math. 27 (2016), 991-1002.

[11] G. Moran, On scattered compact ordered sets, Proc. Amer. Math. Soc. 75 (1979), $355-360$.

[12] A. Ostaszewski, A characterization of compact, separable, ordered spaces, J. London Math. Soc. 7 (1974), 758-760.

[13] Z. Semadeni, Banach Spaces of Continuous Functions, PWN-Polish Sci. Publ., Warszawa, 1971.

[14] D. Yost, The Johnson-Lindenstrauss space, Extracta Math. 12 (1997), 185-192.

[15] V. Zizler, Nonseparable Banach spaces, in: Handbook of the Geometry of Banach Spaces, Vol. 2, North-Holland, Amsterdam, 2003, 1743-1816.

Artur Michalak

Faculty of Mathematics and Computer Science

A. Mickiewicz University in Poznań

Umultowska 87

61-614 Poznań, Poland

E-mail: michalak@amu.edu.pl 